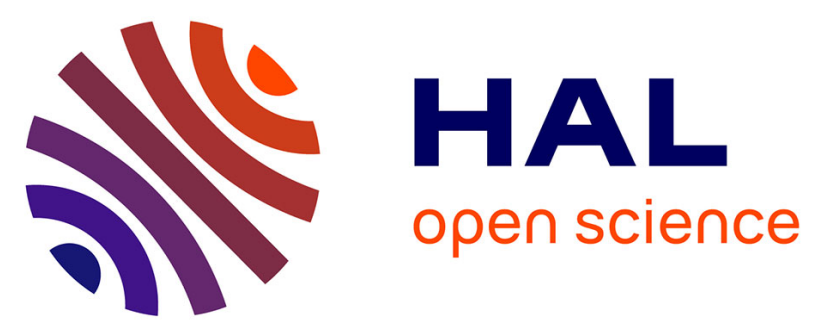

\title{
Defect in the nuclear pore membrane glycoprotein 210-like gene is associated with extreme uncondensed sperm nuclear chromatin and male infertility: a case report
}

Karim Arafah, Fabrice Lopez, Caroline Cazin, Zine-eddine Kherraf, Virginie

Tassistro, Anderson Loundou, Christophe Arnoult, Nicolas Thierry-Mieg, Philippe Bulet, Marie Roberte Guichaoua, et al.

\section{- To cite this version:}

Karim Arafah, Fabrice Lopez, Caroline Cazin, Zine-eddine Kherraf, Virginie Tassistro, et al.. Defect in the nuclear pore membrane glycoprotein 210-like gene is associated with extreme uncondensed sperm nuclear chromatin and male infertility: a case report. Human Reproduction, 2021, 36 (3), pp.693-701. 10.1093/humrep/deaa329 . hal-02985525

\section{HAL Id: hal-02985525 \\ https://hal.science/hal-02985525}

Submitted on 15 Feb 2021

HAL is a multi-disciplinary open access archive for the deposit and dissemination of scientific research documents, whether they are published or not. The documents may come from teaching and research institutions in France or abroad, or from public or private research centers.
L'archive ouverte pluridisciplinaire HAL, est destinée au dépôt et à la diffusion de documents scientifiques de niveau recherche, publiés ou non, émanant des établissements d'enseignement et de recherche français ou étrangers, des laboratoires publics ou privés.

$$
\text { Copyright }
$$




\title{
Defect in the nuclear pore membrane glycoprotein 210 -like gene is associated with extreme uncondensed sperm nuclear chromatin and male infertility: a case report
}

\author{
Karim Arafah ${ }^{1, *}$, Fabrice Lopez ${ }^{2}$, Caroline Cazin ${ }^{3,4}$, \\ Zine-Eddine Kherraf $f^{3,4}$, Virginie Tassistro ${ }^{5}$, Anderson Loundou ${ }^{6}$, \\ Christophe Arnoult ${ }^{3}$, Nicolas Thierry-Mieg ${ }^{7}$, Philippe Bulet ${ }^{1,8}$, \\ Marie-Roberte Guichaoua ${ }^{5}$, and Pierre F. Ray ${ }^{3,4}, *$
}

\begin{abstract}
'Plateforme BioPark d'Archamps, Archamps, France ${ }^{2}$ INSERM U1090, Marseille, France ${ }^{3}$ Institute for Advanced Biosciences, CR Inserm U1209, CNRS UMR5309, University Grenoble Alpes, Team « Génétique, Épigénétique et Thérapies de l'infertilité », Grenoble, France ${ }^{4} \mathrm{CHU}$ Grenoble Alpes, UM GI-DPI, Grenoble 38000, France ${ }^{5}$ Aix Marseille Université, Avignon Université, CNRS, IRD, IMBE, Marseille, France ${ }^{6}$ Department of Public Health, Faculty of Medicine, Methodological Assistance Unity for Clinical Research, Marseille, France ${ }^{7}$ Univ. Grenoble Alpes, CNRS, TIMC-IMAG/BCM, Grenoble 38000, France ${ }^{8}$ Institute for Advanced Biosciences, CR Inserm UI209, CNRS UMR5309, University Grenoble Alpes, Team « Immunologie Analytique des Pathologies Chroniques », Grenoble, France
\end{abstract}

*Correspondence address. Pierre F. Ray, Institute for Advanced Biosciences, CR Inserm UI 209, CNRS UMR5309, University Grenoble Alpes, Team « Génétique, Épigénétique et Thérapies de l'infertilité », Grenoble, France. E-mail: pray@chu-grenoble.fr (P.F.R.) or Karim Arafah, Plateforme BioPark d'Archamps, Bât. Le Forum I, ArchParc, Archamps, France. E-mail: karim.arafah@biopark-archamps.org (K.A.)

\begin{abstract}
After the two meiotic divisions, haploid round spermatids undergo dramatic changes to become mature spermatozoa. One of the main transformations consists of compacting the cell nucleus to confer the sperm its remarkable hydrodynamic property and to protect its DNA from the oxidative stress it will encounter during its reproductive journey. Here, we studied an infertile subject with low sperm count, poor motility and highly abnormal spermatozoa with strikingly large heads due to highly uncondensed nuclear sperm DNA. Whole-exome sequencing was performed on the subject's DNA to identify the genetic defect responsible for this severe sperm anomaly. Bioinformatics analysis of exome sequence data uncovered a homozygous loss of function variant, ENST00000368559.7:c.7|8-IG>A, altering a consensus splice site expected to prevent the synthesis of the nucleoporin 210 like (NUP2 IOL) protein. High-resolution mass spectrometry of sperm protein extracts did not reveal any NUP2IOL peptide sequence in the patient's sperm, contrary to what was observed in control donors, thus confirming the absence of NUP2IOL in the patient's sperm. Interestingly, homozygous Nup2I0I knock-out mice have been shown to be infertile due to a reduced sperm count, a high proportion of round-headed sperm, other head and flagella defects and a poor motility. NUP2IOL is almost exclusively expressed in the testis and sequence analogy suggests that it encodes a nuclear pore membrane glycoprotein. The protein might be crucial to regulate nuclear trafficking during and/or before spermiogenesis, its absence potentially impeding adequate nuclear compaction by preventing the entry of histone variants/transition proteins/protamines into the nucleus and/or by preventing the adequate replacement of core histones. This work describes a new gene necessary for male fertility, potentially improving the efficiency of the genetic diagnosis of male infertility. The function of NUP2IOL still remains to be resolved and its future investigation will help to understand the complex mechanisms necessary for sperm compaction.
\end{abstract}

Key words: male infertility / uncondensed sperm nucleus / chromatin remodelling / spermatogenesis / NUP2 IOL / nucleoporin 210 like / exome sequencing, proteomics 


\section{Introduction}

Genetic defects are believed to be responsible for almost all of the most severe sperm defects, such as azoospermia or severe morphological defects (Coutton et al., 2015; Mitchell et al., 2017; Ray et al., 2017), and genetic studies have elucidated the molecular basis of many specific morphological defects, such as macrozoospermia (Dieterich et al., 2007), globozoospermia (Harbuz et al., 20I I; Koscinski et al., 20II), acephalic spermatozoa (Zhu et al., 20।6; Elkhatib et al., 2017) and multiple morphological anomalies of the flagella (Ben Khelifa et al., 2014; Touré et al., 2020). In recent years, the use of whole-exome sequencing greatly increased the pace of discovery and identified many more genes, especially for the phenotype associated with the multiple morphological anomalies of the flagella (Touré et al., 2020). Here, we identified one patient (PI) with oligoasthenoteratozoospermia but presenting a majority of spermatozoa with strikingly large sperm heads owing to highly uncondensed nuclear sperm DNA. Abnormal sperm with poorly condensed DNA have been described in the past in several sub-phenotypes (Perdrix et al., 201 I; Boitrelle et al., 2013, 2015; Hosseinifar et al., 2015; Yassine et al., 20I5) but the described sperm heads did not present the massive expansion observed in PI. Defects in the aurora kinase $C$ (AURKC) gene were also shown to be responsible for macrozoospermia (Dieterich et al., 2007); however, the enlarged size of the produced spermatozoa was not due to chromatin loosening but to a failed cytokinesis responsible for the presence of four haploid genomes in each sperm head (Dieterich et al., 2009). Here, most of PI's spermatozoa are round but the DNA is extremely uncondensed with a size which largely exceeds that of the round or decondensed spermatozoa described so far. We, therefore, believe that the observed sperm defect described in this manuscript is different from the morphological anomalies described previously in the scientific literature.

We performed whole-exome sequencing on genomic DNA and a proteomic analysis of the spermatozoa in order to identify the molecular events responsible for $\mathrm{PI}$ sperm defects.

\section{Case report}

\section{Patient information and semen analysis}

$\mathrm{PI}$ and his wife, 32 and 30 years old, respectively, had a 5-year period of common primary infertility and consulted the centre for medically assisted reproduction at the Conception Hospital (Marseille, France). They were both childless, with no history of significant illness, normal karyotype and hormonal assessments. PI had no $\mathrm{Y}$ chromosome deletion and a testicular volume within the normal range. The husband is one of six siblings born from first-cousin parents (Supplementary Fig. SI). Before consulting in our centre, the couple underwent ICSI, but with complete fertilization failure. We collected two semen samples from PI, 3 months apart, and one sample from two control donors. Samples were collected by masturbation after 3 days of sexual abstinence. The semen samples were incubated for $30 \mathrm{~min}$ at $37^{\circ} \mathrm{C}$ for liquefaction and then analysed within 30-60 min after collection. The semen parameters were studied on fresh sperm according to the World Health Organization standard methods (Cooper et al., 20l0). The patient and the control donors signed an informed consent permitting the analysis and storage of samples and data into the GERMETHEQUE Biobank (BB-0033-0008I, France). DNA extraction and management were performed at the centre for biological resources (tissues and cells), in the Department of Medical Genetics (La Timone Hospital, Marseille, France). Following sperm analysis, the couple was informed that the ICSI would not be attempted and that they should consider using donor sperm or adoption. The patient did not wish his family to be informed of his condition and did not agree to donate any additional biological samples. The couple was guided to the Centre d'Etude et de Conservation des Fufs et du Sperme (Provence-Alpes-Côte d'Azur Marseille, France) for sperm donation. Sperm parameters from the patient and controls are listed in Table I. In $\mathrm{PI}$, volume and $\mathrm{pH}$ were normal in both semen analyses. Control donors had normal semen parameters. Direct light microscopy analysis revealed severe oligoasthenoteratozoospermia in $\mathrm{PI}$. There were no motile spermatozoa and no spermatozoa with a normal morphology. Shorr staining revealed normal spermatozoa in controls (Fig. IA) and the presence only in $\mathrm{PI}$ of abnormal spermatozoa without an acrosome and presenting very large heads in $81 \%$ and $85 \%$ of the analysed spermatozoa in the two ejaculates, respectively (Fig. IB). In both ejaculates from $\mathrm{PI}$, numerous isolated heads ( $50 \%$ and $33 \%$, respectively) and isolated flagella were observed but numerous clusters of isolated tails made it difficult to establish an accurate percentage. Normal-sized spermatozoa ( $9 \%$ and $5 \%$ ) had a morphologically normal tail but no acrosome. All sperm nuclei were heavily stained with aniline blue (Fig. IC). The DAPI staining revealed chromatin abnormalities evidenced by an uneven colouring with a fleecy appearance in large and normal-sized sperm heads (Fig. ID).

\section{Immunofluorescence}

Immunofluorescence staining was performed on frozen sperm samples fixed with $2 \%$ paraformaldehyde for immunocytology and stained with a human anti-SCP3 (synaptonemal complex protein 3; kindly provided by Christa Heyting) and used at I/250 and revealed by fluorescein isothiocyanate (FITC)-conjugated anti-human IgG diluted at I/I60 in PBS for I h at $37^{\circ} \mathrm{C}$ (Metzler-Guillemain and Guichaoua, 2000). Two control donors with normal semen parameters were used for the immunofluorescent analyses. Two hundred spermatozoa and meiotic cells were analysed for $\mathrm{PI}$, and 500 were analysed for each control donor. Statistical analysis was performed using PASW Statistics Version (IBM SPSS Inc., Chicago, IL, USA). Comparison of the percentages was performed using the $\chi^{2}$ test.

Immunofluorescence staining demonstrated a significantly different number of spermatocytes $(P<0.00 \mathrm{I})$ blocked in first meiotic prophase in $\mathrm{PI}$ (I5.5\%) compared to what was observed in controls $(0.5 \%)$. Regarding the other cells, anti-SCP3 fixed non-specifically to proteins, revealing isolated tails (Fig. IE) and tail-less heads (Fig. IF) from PI ejaculate. In large tail-less heads, the implantation fossa often presented an abnormally concave aspect (Fig. IF). PI sperm analysis showed no staining in the post-nuclear cap region, regardless of the size (Fig. IF and H), contrary to what was observed on the majority of control spermatozoa (Fig. IG), thus evidencing the absence of an acrosome on PI's spermatozoa. In addition, in large-headed spermatozoa, the uncondensed chromatin was observed to spread around the proximal part of the tail (Fig. II). 
Table I Semen parameters from the two ejaculates of the patient PI (Sample I, Sample 2) and of the two control donors used in the study.

\begin{tabular}{|c|c|c|c|c|c|}
\hline & Patient Sample I & Patient Sample 2 & Control I & Control 2 & $\mathbf{N}$ \\
\hline Volume (ml) & 3.7 & 2.5 & 2 & 2.6 & $\geq 1.5$ \\
\hline $\mathrm{pH}$ & 8.3 & 8.5 & 8.3 & 8.3 & $7-8.5$ \\
\hline Sperm count $(\mathrm{M} / \mathrm{ml})$ & 5.6 & 5 & 175 & 110 & $\geq 15$ \\
\hline Progressive motility (\%) & 0 & 0 & 30 & 40 & $\geq 30$ \\
\hline Vitality (\%) & 0 & 0 & 85 & 76 & $\geq 58$ \\
\hline Typical forms (\%) & 0 & 0 & 68 & 51 & $\geq 4$ \\
\hline Round cells $(\mathrm{M} / \mathrm{ml})$ & 5.8 & 5 & 8.4 & 9.8 & \\
\hline Tail-less heads (\%) & 50 & 33 & 2 & 0 & \\
\hline Isolated tails (\%) & 12 & 27 & 6 & 17 & \\
\hline Abnormal tails (\%) & 11 & 6 & 7 & 2 & \\
\hline Normal sized (\%) & 9 & 5 & 98 & 99 & \\
\hline Large-headed spermatozoa (\%) & 81 & 85 & 0 & 0 & \\
\hline
\end{tabular}

Normal values $(\mathrm{N})$ are indicated as defined by the World Health Organization (Cooper et al., 20I0). The percentages of isolated tails in Patient I do not take into account the clusters of isolated tails in which the number of tails is impossible to evaluate.

\section{Whole-exome sequencing}

Genomic DNA was isolated from EDTA blood using the DNeasy Blood \& Tissue Kits from QIAGEN SA (Courtaboeuf, France). Genetic data were obtained on the NGS facility of Grenoble Alpes Hospital, at the Institute of Biology and Pathology. Coding regions and intron/exon boundaries were sequenced after enrichment using the Truseq Exome vI.2 (Illumina, San Diego, CA, USA) at the Grenoble Teaching Hospital molecular platform on an Illumina NextSeq 550 system. Exomes data were analysed using a bioinformatics pipeline developed in-house using two modules, both distributed under the GNU General Public License v3.0 and available on github: https://github.com/ntm/grexome-TIMC-Primary and https://github.com/ntm/grexome-TIMC-Secondary and as described, in part, in Martinez et al. (2020). Variants with a minor allele frequency greater than $1 \%$ in gnomAD v2.0, $3 \%$ in 1000 Genomes Project phase 3, or 5\% in NHLBI ESP6500, were filtered out and only variants predicted to have high-impact (e.g. stop-gain or frameshift variants) by variant Effect Predictor v92 (McLaren et al., 2016) were scrutinized. Ninety-three percent of PI's targeted sequences (defined as all coding exons belonging to ENSEMBL canonical transcripts plus 10 flanking intronic nucleotides on each side) had a sequencing depth of at least 10x.

After filtering out, only four hemizygous or bi-allelic variants were predicted by Variant Effect Predictor to have a highly deleterious impact (Table II). Two contiguous variants affected the insulin like growth factor binding protein like I gene (IGFBPLI) described to be associated with Glaucomatocyclitic Crisis and Vitreous Detachment. IGFBPLI appears to be ubiquitously expressed in both GTEx and Illumina bodymap 2 (Supplementary Fig. S2A and B) and has no connection with sperm production. Another variant was found in the spermidine/ spermine $\mathrm{NI}$-acetyl transferase-like I (SATLI) gene. SATLI, located on the $X$ chromosome, is described in GTEx to be over-expressed in the testis but with a low expression level (2 transcripts per million). In other databases, its expression is also weak but is ubiquitous
(Supplementary Fig. S2C and D). As reported in GnomAD (https:// gnomad.broadinstitute.org/), the identified variant, ENSP00000425 421. I: :.Trp442Ter, is found in the general population with a frequency of $0.26 \%$ (Table II). The gene's low testis expression and the variant's high frequency are not compatible with the severity and rarity of the studied phenotype. The last homozygous variant, c.7/8-IG $>A$ (Fig. 2A and B), affects the nucleoporin 210 like gene (NUP2 IOL) coding for the nuclear pore membrane glycoprotein 210-like (Q5VU65 in UniProtKB, hereafter referred to as NUP2 IOL). This glycoprotein has an unknown function and is almost exclusively expressed in the testis (Fig. 2C and Supplementary Fig. S2E and F). Owing to its high and specific expression, NUP2 IOL appeared as the best candidate to explain the patient's sperm anomaly.

\section{Data on NUP2 IOL and variant c.7 |8-|G>a}

The identified variant, ENST00000368559.7:c.7I 8-IG>A (Fig. 2A) is absent from the gnomAD database, consistent with the rarity of the identified phenotype. The altered nucleotide affects the consensus acceptor site of NUP2 IOL exon 6 (Fig. 2B) and is predicted by NetGene2 (http://www.cbs.dtu.dk/services/NetGene2/) to induce the skipping of exon 6, resulting in a premature stop codon. As the event does not occur in the last coding exon, the altered RNA is expected to be degraded by nonsense-mediated mRNA decay (Nagy and Maquat, 1998) and any remaining transcripts would not produce a functional protein. Unfortunately, PI did not want to provide additional samples for research and we could not perform RT-PCR to precisely assess the effect of the variant on NUP2/OL splicing.

The NUP2IOL variant appeared as homozygous following both exome sequencing and Sanger sequencing. As we could not follow its familial segregation, we cannot exclude the possibility that it might be hemizygous with a heterozygous deletion in the counter allele. Given the consanguinity in this family, it is more likely that the observed results come from a homozygous mutation. 

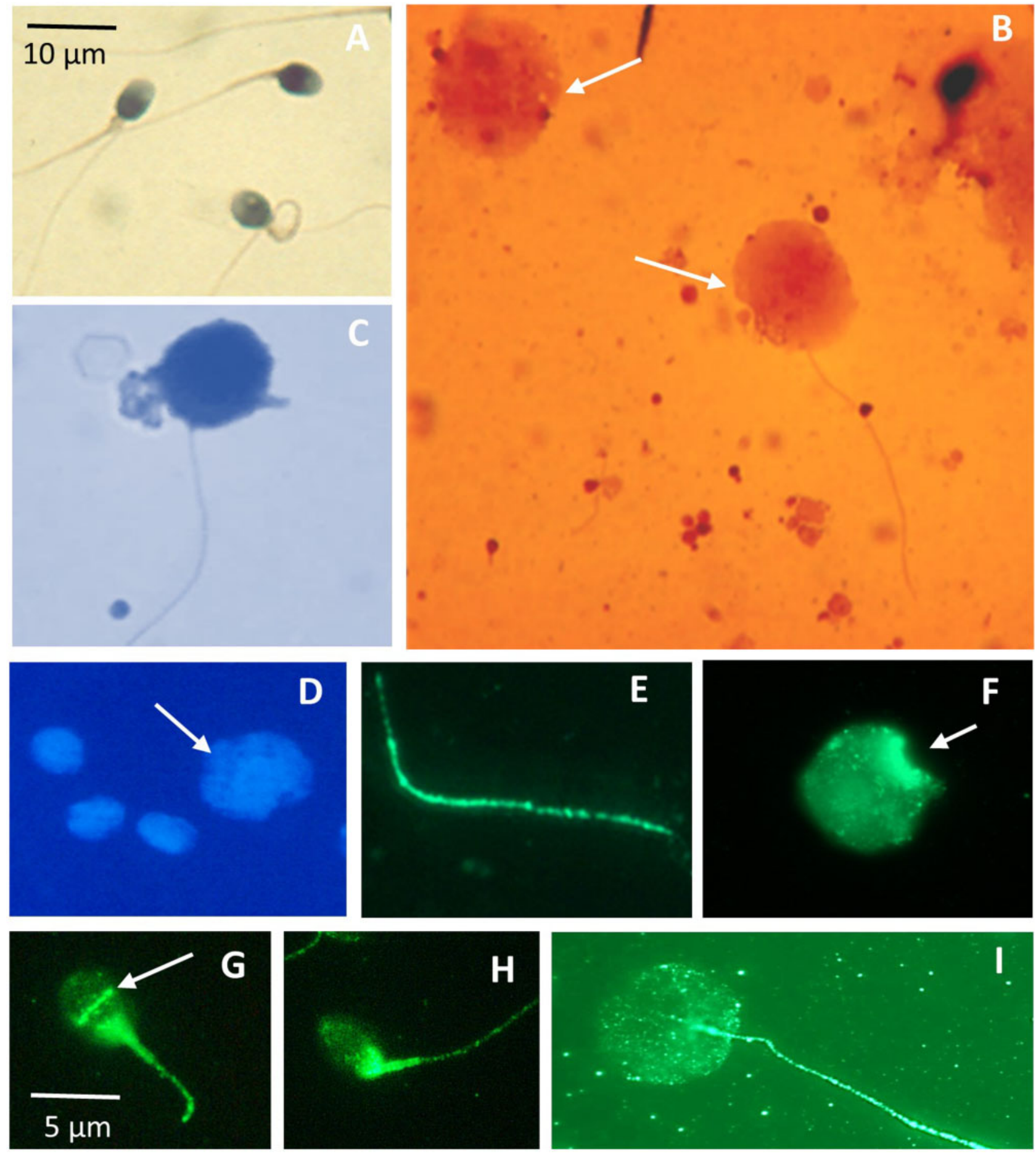

Figure I. Morphological observations of the uncondensed sperm from patient I after histological staining and immunofluorescence. (A) Normal spermatozoa from the control donors with the Shorr staining. (B) Morphological aspect of the uncondensed chromatin spermatozoa from the patient (PI) with the Shorr staining (bottom arrow: head with tail, top arrow: tail-less head). (C) Aniline blue staining showing heavily stained uncondensed chromatin from PI. (D) DAPI staining showing fleecy appearance in large (arrow) and normal-sized sperm head from the patient. (E-I) Immunofluorescence staining with FITC-conjugated anti-synaptonemal complex protein 3 antibody: (E) Isolated tail. (F) Isolated head showing the abnormal concavity of the implantation fossa (arrow) in an uncondensed chromatin spermatozoa. (G) Post-nuclear cap region in spermatozoa from the control donors (arrow). $(\mathrm{H})$ Post-nuclear cap region failed in the normal-sized spermatozoa of the patient. (I) Uncondensed nuclear chromatin surrounding the proximal part of the tail in the patient sperm. A-F and I: scale bar $=10 \mu \mathrm{m} ; \mathrm{G}$ and $\mathrm{H}$ : scale bar $=5 \mu \mathrm{m}$. 
Table II List of all homozygous and hemizygous deleterious variants identified in patient I.

\begin{tabular}{|c|c|c|c|c|c|}
\hline Symbol & Position & HGVSc. & HGVSp. & gnomAD & GTEX \\
\hline NUP2IOL & chrl:154I38239 & ENST00000368559.7:c.7| 8-IG>A & & 0 & 41.0 \\
\hline IGFBPLI IGFBPLI & chr9:384| | 406 & ENST00000377694.2:c.824_830del & ENSP00000366923.I:p.Asp275AlafsTer I5 & $8.0 \mathrm{E}-3$ & 2.1 \\
\hline IGFBPLI IGFBPLI & chr9:384||4|8 & ENST00000377694.2:c.8I8_8I9insA & ENSP00000366923. I:p.Pro274SerfsTer3 & $1.8 \mathrm{E}-3$ & 2.1 \\
\hline SATLISATLI & chrX:85I07644 & ENST0000050923I.I:c.I325G>A & ENSP0000042542I.I:p.Trp442Ter & $2.6 \mathrm{E}-3$ & 6.0 \\
\hline
\end{tabular}

gnomAD, the frequency of the variant as indicated in gnomAD v2.2.I (https://gnomad.broadinstitute.org/); GTEX, the ratio of testis expression over the averaged expression of the gene in all other organs. Expression values are extracted from GTEX database (https://gtexportal.org/home/). HGVSc and HGVSp, the expected sequence modifications according to Human Genome Variation Society (HGVS) in regards to the cDNA sequence (c.) and to the protein sequence (p.) of the corresponding genes.

IGFBPLI: insulin like growth factor binding protein like I; NUP2 IOL, nucleoporin 210 like; SATLI, spermidine/spermine $\mathrm{nI}$-acetyl transferase-like I.

\section{Bottom-up proteomics analysis}

Proteins were extracted from $\sim 1.5$ million spermatozoa from both $\mathrm{PI}$ and control subjects, as previously described (de Yebra and Oliva, 1993). Bottom-up proteomics-based mass spectrometry was performed, as described previously (Masson et al., 2018; Arafah et al., 2020). Briefly, tandem mass spectrometry analyses were carried out using a nanoHPLC (Ultimate 3000, Thermo Scientific, Germany) coupled with a high-resolution mass spectrometer (Q-Exactive, Thermo Scientific, Germany) using a biphasic linear gradient from $2 \%$ to $45 \%$ of acetonitrile $/ 0.1 \%$ formic acid for $108 \mathrm{~min}$, and from $45 \%$ to $80 \%$ of acetonitrile $/ 0.1 \%$ formic acid for 4 min., finishing with a plateau at $80 \%$ for $10 \mathrm{~min}$. An exclusion list of the seven most representative ions from the trypsin autolysis was added to the method. To investigate the protein content in $\mathrm{PI}$ and control sperm, extensive sequencing was performed using the Sequest HT proteomics database search engine and against the human proteome housed in the UniProtKB database. Peptide identification parameters were set as previously described (Deshoux et al., 2020) with phosphorylation (on serine, threonine and tyrosine residues) set as additional dynamic modifications. Only proteins obtained from peptides with a high confident identification (false discovery rate not exceeding 0.01 ) were considered in this study and we focused on NUP2IOL sequences. Based on bottom-up proteomics sequencing, 12 unique peptides were found to match the human NUP2IOL and were only characterized in control sperm, whereas none of them was retrieved from PI (Table III). Altogether, these unique peptides, which were all characterized with a high level of confidence (Table III), covered $9.27 \%$ of the NUP2IOL sequence. To confirm these results, we also analysed sequence strings with medium or low confidence scores. Again, we did not identify any protein sequence in relation to the NUP2 IOL glycoprotein in PI sperm proteome.

To confirm that the process of protein extraction and identification was comparable between $\mathrm{PI}$ and control, we compared the percentage of identified proteins involved in different biological processes and molecular functions from $\mathrm{PI}$ and control sperm (Supplementary Fig. S3). No differences were observed between theses samples, consistent with the fact that the absence of NUP2 IOL observed in PI is not an artefact caused by technical problems or a low protein quantity.

\section{Discussion}

Here, we identified an infertile man with oligoasthenoteratozoospermia with a majority of spermatozoa presenting a strikingly uncondensed chromatin. A similar phenotype albeit with a more modest decondensation has been described in homozygous mice for the mutant allele of the bromodomain testis associated gene Brdt $\left(B r d t^{\triangle B D I} / \Delta^{A B D}\right)$, lacking the first bromodomain of the protein (Gaucher et al., 20I2). Full knock-out (KO) male mice (with a Brdt null allele) revealed a total absence of post-meiotic cells and round spermatids (Shang et al., 2007; Gaucher et al., 2012). Another hallmark of PI's spermogram was the high number of isolated flagella, as is normally observed in acephalic spermatozoa. A homozygous BRDT mutation has been recently identified by whole-exome sequencing in an infertile patient suffering from acephalic spermatozoa (Li et al., 2017), however, the authors did not provide strong evidence of the pathogenicity of the identified BRDT missense variant (p.Gly928Asp). We thus think that BRDT is unlikely to be associated to acephalic spermatozoa, especially as the presence of uncondensed sperm heads has never been mentioned as an additional marker of acephalic spermatozoa (Chemes et al., 1999; Zhu et al., 2016; Elkhatib et al., 2017; Shang et al., 2018). Enlarged spermatozoa have also been linked to genetic defects in the AURKC gene (Dieterich et al., 2007, 2009), but again with a different phenotype than what was observed in PI. Finally, PI exome data did not identify any deleterious variants in BRDT or AURKC, ruling out the involvement of these genes in PI's infertility.

Exome sequencing was performed on $\mathrm{PI}$ to identify the gene defect responsible for the $\mathrm{PI}$ sperm anomaly. No deleterious variants were identified in any gene previously described to be associated with infertility. We identified a homozygous truncating variant in NUP2/OL, a gene of unknown function expressed almost exclusively in testicular germ cells. Several arguments indicate that the identified variant is responsible for the patient's infertility. The variant, ENST00000368559.7:c.7I8-IG>A, affects a consensus splice acceptor site, is homozygous and is predicted to prevent the production of any functional protein. This prediction was supported by the fact that the NUP2IOL protein was detected in protein extracts from control sperm but not in PI's sperm. Additionally, the c.7I8-IG>A variant was not detected in any of the 141456 subjects analysed in gnomAD v2.I.I, consistent with the rarity of the observed phenotype. Most convincingly, homozygous Nup2 IOI KO mice were infertile owing to oligozoospermia and the presence of spermatozoa with a high proportion of rounded heads and flagellar defects inducing no or poor motility (Walters et al., 2009) and reported by Professor Elizabeth Bryda (personal communication).

Based on sperm morphology, we can hypothesize that the spermatogenic defect in this patient occurs after meiosis when the spermatids 
A

c. $718-1 \mathrm{G}>\mathrm{A}$

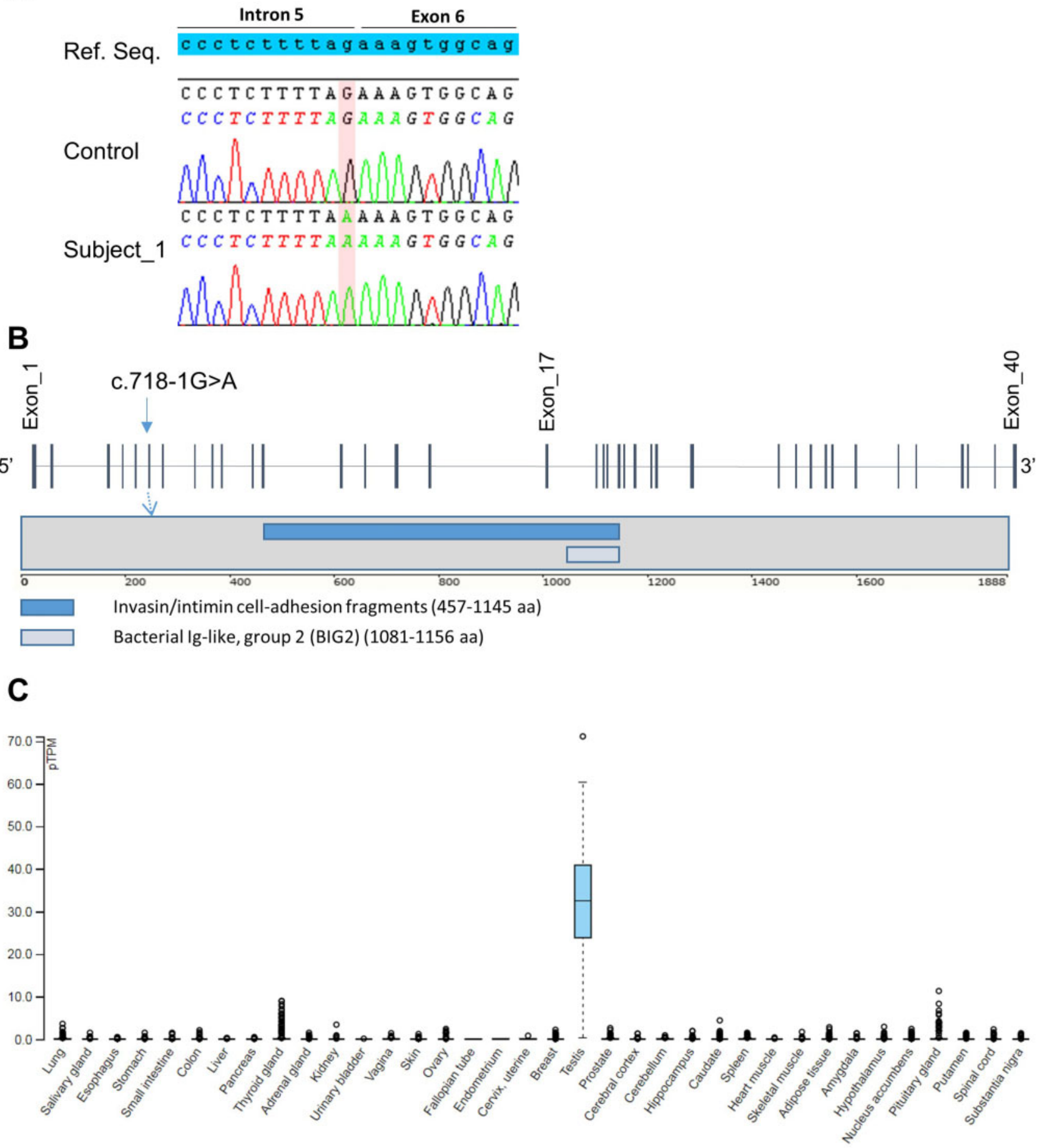

Figure 2. Description of the identified NUP2 IOL variant and gene expression. (A) Electropherograms from Sanger sequencing showing the identified nucleoporin 210 like (NUP2IOL) variant. The substituted nucleotide is highlighted in light pink and is referred to as c.7I8-IG $>$ A. (B) Localization of the variant within the NUP2 IOL gene and protein. (C) Tissue expression of NUP2IOL from GTEX (https://gtexportal.org/home/ gene/NUP2 IOL). Values are expressed as protein-transcripts per million (pTPM). Circles correspond to one independent experiment. Values for testis are expressed as a box plot presenting the 25 th percentile (bottom of the rectangle), the 50th percentile (middle lane) and 75th percentile (top), one point was excluded (hollow circle). 


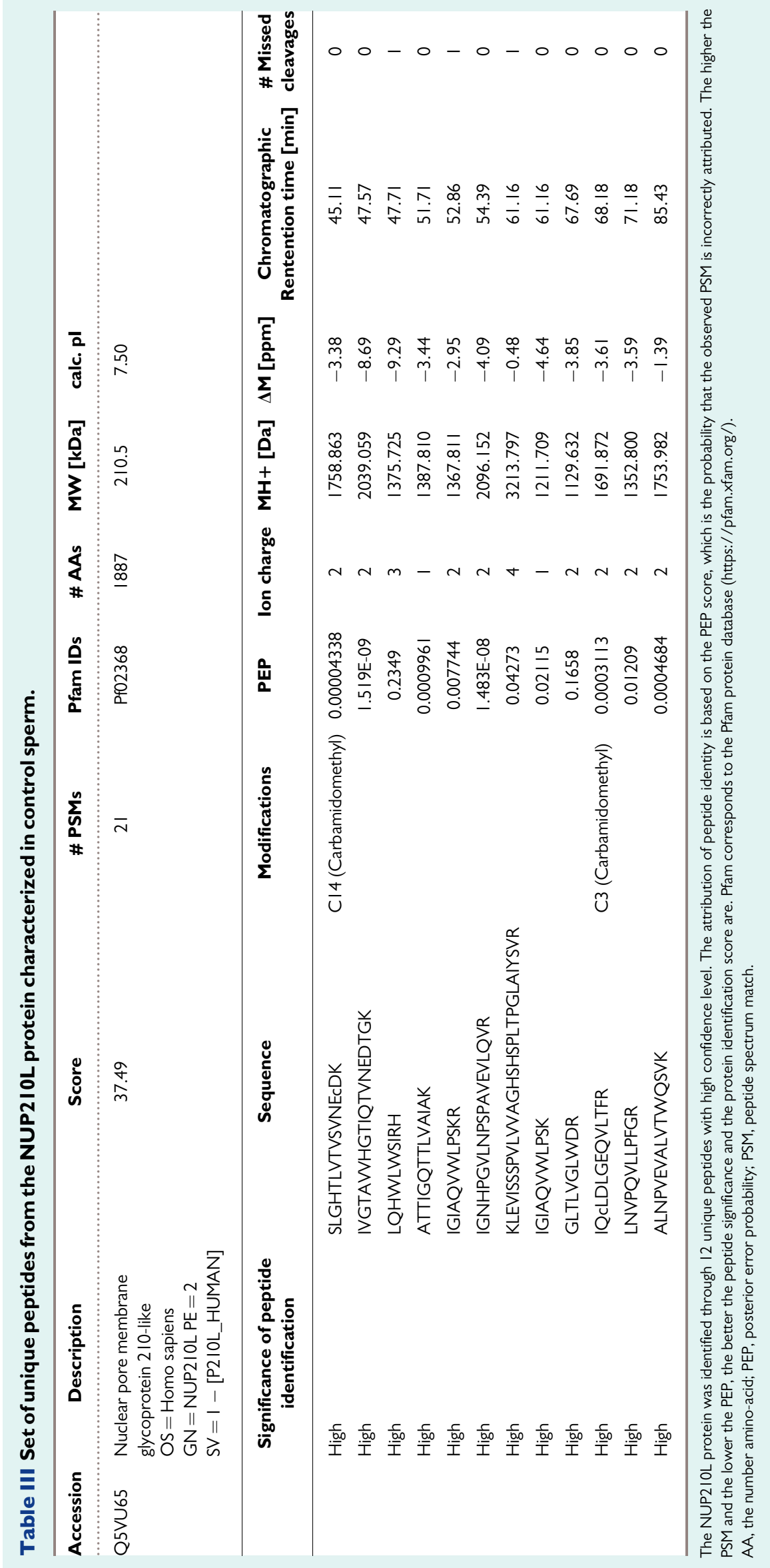


begin their elongation and exchange the majority of their histones for transition proteins. The positive reaction with aniline blue staining in all sperm proves the presence of a high percentage of histones in all these nuclei and the highly uncondensed chromatin of the sperm suggests the presence of highly abnormal nucleosomic structures. Interestingly, all spermatozoa were stained by a vital stain (eosin-nigro$\sin$ ) and scored as non-viable. This could be caused by the important nuclear expansion contributing to the stretching of the cell membrane, allowing the sperm to take up the stain. In addition, numerous flagellar defects and flagella-less spermatozoa were observed in $\mathrm{PI}$ and in Nup2 I OI KO mice. Flagella formation is concomitant with nuclear condensation and the flagellum needs to be strongly attached to the nucleus via several proteins, among which is SadI And UNC84 Domain Containing 5 (SUN5), involved in headless spermatozoa syndrome (Shang et al., 20I8). Here, it is possible that the nucleus' structure is so severely altered that the adequate attachment of the flagella is no longer possible, thus explaining the important flagellar defects observed in $\mathrm{PI}$ and $\mathrm{KO}$ mice. Last, in view of the severity of the defect, ICSI is unlikely to be successful for men with NUP2 IOL deleterious variants.

In mice, Nup210l is described as only expressed in male germ cells with an initial and strong expression in primary spermatocytes (Supplementary Fig. 2E-G), and data from proteinatlas (https://www. proteinatlas.org/ENSG00000 I 43552-NUP2 IOL/tissue/testis\#). This expression profile is consistent with what is observed in Nup2 IOI KO mice, which present a severe pre-meiotic and meiotic blockage (Walters et al., 2009). Our patient also had a low sperm count ( 5 million/ml) and numerous primary spermatocytes were observed in the ejaculate. These observations suggest that the absence of NUP2IOL, in human and mouse, induces a partial arrest during the first meiotic division. Some germ cells then seem to overcome this meiotic block and pursue their differentiation but with a highly abnormal chromatin compaction and flagellar formation.

Sequence analogy suggests that NUP2 IOL encodes a nuclear pore membrane glycoprotein. The protein might be crucial to regulate nuclear-cytoplasmic trafficking during and/or before spermiogenesis. Its absence potentially alters nuclear compaction by preventing the entry of histone variants/transition proteins/protamines into the nucleus and/or by preventing the adequate removal of core histones. Interestingly, KO male mice exhibit Sertoli cell degeneration, which may also be at the origin of the sperm morphological defects observed in affected mice and PI. This degenerative process may impair normal Sertoli cells — spermatids interactions by perturbing nucleocytoplasmic trafficking and sperm differentiation.

In summary, our study describes a severe sperm anomaly in which the nuclear chromatin of spermatozoa is highly uncondensed. We revealed that this anomaly is likely caused by a genetic defect in NUP2 IOL, coding for a nuclear pore membrane glycoprotein of unknown function and almost solely expressed in male germ cells. Our observations and the previously published data suggest that NUP2IOL is critical to prepare sperm nuclear condensation as early as meiosis I and that its action, albeit potentially indirect, extends until stages 12 14 of mouse spermiogenesis when transition proteins and protamines intervene to induce nuclear condensation. Additional work on wildtype and $\mathrm{KO}$ mice is critical to characterize the precise role of NUP2 IOL in meiosis and/or spermiogenesis.

\section{Data availability}

The data underlying this article will be shared on reasonable request to the corresponding author.

\section{Acknowledgements}

We are grateful to Dr. Saadi Khochbin from the Grenoble Institute for Advanced Biosciences and Pr. Elizabeth Bryda from the University of Missouri, for fruitful exchanges. We thank D. Rousseau, C. Metton, M.J. Fays-Bernardin and D. Daioglou for technical assistance. Genetic data were obtained on the NGS facility of Grenoble Alpes Hospital, at the Institute of Biology and Pathology.

\section{Authors' roles}

Conceived and designed the study: M.R.G., K.A., P.B. and P.R.; designed the proteomics study and analysed the data: K.A.; designed and analysed exome data: P.R. and N.T.M.; technical assistance: V.T. and A.L.; performed the gene variant analyses: P.R., Z.E.K. and C.C.; drafted the manuscript: M.R.G., K.A., P.B., P.R. All authors interpreted the data.

\section{Funding}

This work was partly funded by the FLAGEL-OME project of the French National Research Agency. Proteomics studies were funded by the R\&D budget from the BioPark Platform of Archamps.

\section{Conflict of interest}

The authors declare that there is no conflict of interest.

\section{References}

Arafah K, Kriegsmann M, Renner M, Lasitschka F, Fresnais M, Kriegsmann K, Winterfeld M. V, Goeppert B, Kriegsmann J, Casadonte $\mathrm{R}$ et al. Microproteomics and immunohistochemistry reveal differences in Aldo-Keto reductase family I member C3 in tissue specimens of ulcerative colitis and Crohn's disease. Prot Clin Appl 2020; I 4: el 9001 I0.

Ben Khelifa M, Coutton C, Zouari R, Karaouzène T, Rendu J, Bidart M, Yassine S, Pierre V, Delaroche J, Hennebicq S et al. Mutations in DNAHI, which encodes an inner arm heavy chain dynein, lead to male infertility from multiple morphological abnormalities of the sperm flagella. Am J Human Genet 20I4;94:95-104.

Boitrelle F, Albert M, Petit J-M, Ferfouri F, Wainer R, Bergere M, Bailly M, Vialard F, Selva J. Small human sperm vacuoles observed under high magnification are pocket-like nuclear concavities linked to chromatin condensation failure. Reprod Biomed 2013;27: 20I-2II. 
Boitrelle F, Pagnier M, Athiel Y, Swierkowski-Blanchard N, Torre A, Alter L, Muratorio C, Vialard F, Albert M, Selva J. A human morphologically normal spermatozoon may have noncondensed chromatin. Andrologia 2015;47:879-886.

Chemes HE, Puigdomenech ET, Carizza C, Olmedo SB, Zanchetti F, Hermes R. Acephalic spermatozoa and abnormal development of the head-neck attachment: a human syndrome of genetic origin. Hum Reprod 1999; 14:1811-1818.

Cooper TG, Noonan E, Eckardstein S. V, Auger J, Baker HWG, Behre HM, Haugen TB, Kruger T, Wang C, Mbizvo MT et al. World Health Organization reference values for human semen characteristics. Hum Reprod Update 2010; 1 6:231-245.

Coutton C, Escoffier J, Martinez G, Arnoult C, Ray PF. Teratozoospermia: spotlight on the main genetic actors in the human. Hum Reprod Update 2015;2 I :455-485.

Deshoux M, Masson V, Arafah K, Voisin S, Guschinskaya N, Munster MV, Cayrol B, Webster CG, Rahbé Y, Blanc S et al. Cuticular structure proteomics in the pea aphid Acyrthosiphon pisum reveals new plant virus receptor candidates at the tip of maxillary stylets. J Proteome Res 2020; 19:1319-1337.

Dieterich K, Soto Rifo R, Karen Faure A, Hennebicq S, Amar BB, Zahi M, Perrin J, Martinez D, Sèle B, Jouk P-S et al. Homozygous mutation of AURKC yields large-headed polyploid spermatozoa and causes male infertility. Nat Genet 2007;39:66I-665.

Dieterich K, Zouari R, Harbuz R, Vialard F, Martinez D, Bellayou H, Prisant N, Zoghmar A, Guichaoua MR, Koscinski I et al. The Aurora Kinase C c.l44delC mutation causes meiosis I arrest in men and is frequent in the North African population. Hum Mol Genet 2009; 1 8: 1301-1309.

Elkhatib RA, Paci M, Longepied G, Saias-Magnan J, Courbière B, Guichaoua M-R, Lévy N, Metzler-Guillemain C, Mitchell MJ. Homozygous deletion of SUN5 in three men with decapitated spermatozoa. Hum Mol Genet 2017;26:3167-3।7I.

Gaucher J, Boussouar F, Montellier E, Curtet S, Buchou T, Bertrand $S$, Hery P, Jounier S, Depaux A, Vitte A-L et al. Bromodomain-dependent stage-specific male genome programming by Brdt. EMBO J 20।2;3 I :3809-3820.

Harbuz R, Zouari R, Pierre V, Ben Khelifa M, Kharouf M, Coutton C, Merdassi G, Abada F, Escoffier J, Nikas $Y$ et al. A recurrent deletion of DPYI9L2 causes infertility in man by blocking sperm head elongation and acrosome formation. Am J Hum Genet 2011;88: $35 \mathrm{I}-36 \mathrm{I}$.

Hosseinifar H, Yazdanikhah S, Modarresi T, Totonchi M, Sadighi Gilani MA, Sabbaghian M. Correlation between sperm DNA fragmentation index and CMA3 positive spermatozoa in globozoospermic patients. Andrology 2015;3:526-53I.

Koscinski I, Elinati E, Fossard C, Redin C, Muller J, Velez de la Calle J, Schmitt F, Ben Khelifa M, Ray PF, Ray P et al. DPY I9L2 deletion as a major cause of globozoospermia. Am J Hum Genet 201।;88: 344-350.

Li L, Sha Y, Wang X, Li P, Wang J, Kee K, Wang B. Whole-exome sequencing identified a homozygous BRDT mutation in a patient with acephalic spermatozoa. Oncotarget 2017;8:199|4-19922.

Martinez G, Beurois J, Dacheux D, Cazin C, Bidart M, Kherraf Z-E, Robinson DR, Satre V, Le Gac G, Ka C et al. Biallelic variants in
MAATSI encoding CFAP9I, a calmodulin-associated and spokeassociated complex protein, cause severe asthenoteratozoospermia and male infertility. J Med Genet 2020;57: 708-716.

Masson V, Arafah K, Voisin S, Bulet P. Comparative proteomics studies of insect cuticle by tandem mass spectrometry: application of a novel proteomics approach to the Pea Aphid Cuticular proteins. Proteomics 2018; 18: 1700368.

McLaren W, Gil L, Hunt SE, Riat HS, Ritchie GRS, Thormann A, Flicek $P$, Cunningham F. The Ensembl variant effect predictor. Genome Biol 2016; I7:122.

Metzler-Guillemain C, Guichaoua MR. A simple and reliable method for meiotic studies on testicular samples used for intracytoplasmic sperm injection. Fertil Steril 2000;74:916-919.

Mitchell MJ, Metzler-Guillemain C, Toure A, Coutton C, Arnoult C, Ray PF. Single gene defects leading to sperm quantitative anomalies. Clin Genet 2017;91:208-216.

Nagy E, Maquat LE. A rule for termination-codon position within intron-containing genes: when nonsense affects RNA abundance. Trends Biochem Sci 1998;23:198-199.

Perdrix A, Travers A, Chelli MH, Escalier D, Do Rego JL, Milazzo JP, Mousset-Siméon N, Macé B, Rives N. Assessment of acrosome and nuclear abnormalities in human spermatozoa with large vacuoles. Hum Reprod 2011;26:47-58.

Ray PF, Toure A, Metzler-Guillemain C, Mitchell MJ, Arnoult C, Coutton C. Genetic abnormalities leading to qualitative defects of sperm morphology or function. Clin Genet 2017;91: 217-232.

Shang E, Nickerson HD, Wen D, Wang X, Wolgemuth DJ. The first bromodomain of Brdt, a testis-specific member of the BET subfamily of double-bromodomain-containing proteins, is essential for male germ cell differentiation. Development 2007; I 34:3507-35I 5.

Shang Y, Yan J, Tang W, Liu C, Xiao S, Guo Y, Yuan L, Chen L, Jiang $H$, Guo $X$ et al. Mechanistic insights into acephalic spermatozoa syndrome-associated mutations in the human SUN5 gene. J Biol Chem 2018;293:2395-2407.

Touré A, Martinez G, Kherraf Z-E, Cazin C, Beurois J, Arnoult C, Ray PF, Coutton $C$. The genetic architecture of morphological abnormalities of the sperm tail. Hum Genet 2020.

Walters EM, Bauer BA, Franklin CL, Evans TJ, Bryda EC, Riley LK, Critser JK. Mutational insertion of a ROSA26-EGFP transgene leads to defects in spermiogenesis and male infertility in mice. Comp Med 2009;59:545-552.

Yassine S, Escoffier J, Martinez G, Coutton C, Karaouzène T, Zouari R, Ravanat J-L, Metzler-Guillemain C, Lee HC, Fissore R et al. Dpyl912-deficient globozoospermic sperm display altered genome packaging and DNA damage that compromises the initiation of embryo development. Mol Hum Reprod 2015;2I: 169-185.

Yebra L. D, Oliva R. Rapid analysis of mammalian sperm nuclear proteins. Anal Biochem 1993;209:201-203.

Zhu F, Wang F, Yang X, Zhang J, Wu H, Zhang Z, Zhang Z, He X, Zhou P, Wei $Z$ et al. Biallelic SUN5 mutations cause autosomalrecessive acephalic spermatozoa syndrome. Am J Hum Genet 2016; 99:942-949. 